\title{
Shaping Single Offset Reflector Antennas Using Local Axis-Displaced Confocal Quadrics
}

\author{
Rafael A. Penchel, ${ }^{1}$ José R. Bergmann, ${ }^{2}$ and Fernando J. S. Moreira ${ }^{3}$ \\ ${ }^{1}$ Department of Electrical Engineering, Federal University of Technology-Paraná, 86300-000 Cornélio Procópio, PR, Brazil \\ ${ }^{2}$ Center for Telecommunications Studies (CETUC), Catholic University of Rio de Janeiro, 22453-900 Rio de Janeiro, RJ, Brazil \\ ${ }^{3}$ Department of Electronics Engineering, Federal University of Minas Gerais, 31270-901 Belo Horizonte, MG, Brazil
}

Correspondence should be addressed to José R. Bergmann; bergmann@cetuc.puc-rio.br

Received 22 June 2016; Revised 20 November 2016; Accepted 4 December 2016

Academic Editor: Giuseppe Mazzarella

Copyright (C) 2016 Rafael A. Penchel et al. This is an open access article distributed under the Creative Commons Attribution License, which permits unrestricted use, distribution, and reproduction in any medium, provided the original work is properly cited.

\begin{abstract}
This work investigates a novel numerical procedure for the solution of an exact formulation for the Geometrical Optics synthesis of a single reflector antenna by simultaneously imposing Snell's Law and Conservation of Energy in a tube of rays, yielding a second-order nonlinear partial differential equation of Monge-Ampère type, which can be solved as a boundary value problem. The investigation explores the interpolating properties of confocal quadrics to locally represent the shaped reflector surface. It allows the partial derivatives involved in the formulation to be analytically expressed. To illustrate the method, two examples of offset single reflectors shaped to radiate a Gaussian power density within a superelliptical contoured beam are presented. The results are validated by Physical Optics analysis with equivalent edge currents.
\end{abstract}

\section{Introduction}

Shaped reflector antennas can be employed to provide an optimized coverage with high gains and low cochannel interference. Several techniques based on Geometrical Optics (GO) have been used for the shaping of single and dual reflector antenna configurations. Among these techniques, [1-4] presented exact formulations for the GO synthesis of single and dual offset shaped reflector configurations by simultaneously imposing Snell's Law and Conservation of Energy in a tube of rays. They lead to second-order nonlinear partial differential equations of Monge-Ampère (MA) type, which can be numerically solved as boundary value problems [1-4]. For the solution, the authors employed a finite-difference scheme associated to regular grids to approximate the derivatives. Then, the linearized form of the MA equation was iteratively solved [4-7]. Alternatively, [8] has proposed a GO synthesis technique for multiple reflectors where the surfaces are locally approximated by a biparabolic expansion and their parameters are obtained by solving linear equations. It has been effectively applied in designs of multiple reflectors to produce a uniform phase in front of an antenna aperture [8]. For circular symmetric geometries, $[9,10]$ presented a GO synthesis scheme based on the combination of conic sections to locally represent the reflector generatrices. Similarly, the conic parameters were also obtained by solving linear equations.

In this work, motivated by the effectiveness of the local representation for shaped reflectors, we explore the use of axis-displaced confocal quadrics into the numerical solution of the MA equation. Instead of using finite-difference schemes to calculate the derivatives at grid points, the surface is locally represented by the confocal quadrics, where their parameters are obtained by interpolation, allowing the analytical representation of the derivatives, together with the simplification of the MA equation. It also allows some flexibility in the definition of the grid points that are used to improve the representation of the reflector surface. To illustrate the numerical scheme, it has been applied to single reflector synthesis, where the reflector surface yields a prescribed far-field radiation pattern when illuminated by a point source (feed horn), as shown in Figure 1.

This work is organized as follows: Section 2 presents the several steps involved in the synthesis technique. The 


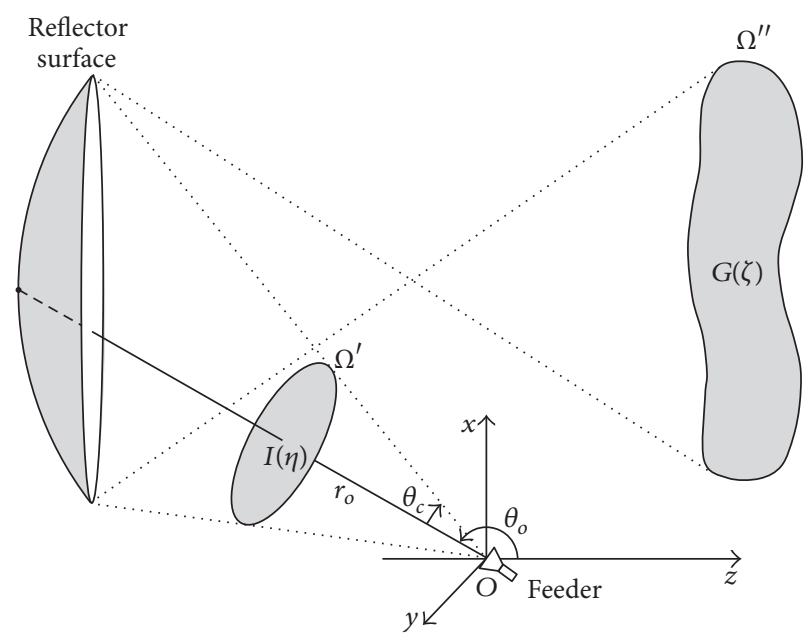

FIGURE 1: Geometry of the offset reflector antenna.

shaped reflector surface is defined by a set of grid points and confocal quadrics that are employed to locally interpolate the surface. The analytical surface derivatives resulting from the interpolation process are replaced into the MA equation and the boundary condition at the reflector's edge leads to a system of nonlinear equations. To solve the system, an iterative numerical technique is employed. Section 3 describes the application of the numerical scheme in the synthesis of two examples of single offset reflector antennas designed to generate a far-field beam with a superelliptical contour. A Physical Optics (PO) analysis plus edge currents technique is employed to verify the synthesized reflector antenna $[11,12]$. The conclusions are presented in Section 4.

\section{GO Shaping Technique}

In the problem illustrated in Figure 1, the cone of rays within the contour $\Omega^{\prime}$ and with radiation pattern $I(\eta)$ is reflected within a beam with contour $\Omega^{\prime \prime}$ where the power density is represented by $G(\zeta)$. It is simpler to use the complex coordinates (stereographic projection) adopted in [2-4] to describe directions. $\eta$ and $\zeta$ are such coordinates and here they represent the incident and reflected ray directions, respectively, defined as

$$
\begin{aligned}
& \eta=\cot \left(\frac{\theta}{2}\right) e^{i \phi}, \\
& \zeta=\cot \left(\frac{\alpha}{2}\right) e^{i \beta},
\end{aligned}
$$

where $\theta, \phi$ and $\alpha, \beta$ are the spherical angular directions of the incident and reflected rays, respectively, as shown in Figure 2. Two auxiliary coordinate systems are used to describe ray directions: primed $\left(^{\prime}\right)$ and double primed $\left({ }^{\prime \prime}\right)$, related to feeder and far-field, respectively. The function $r(\eta)$ describes the distance of the reflector point at $\eta$ to the origin and $L(\eta)$ is a real function that represents the reflector's surface [2-4]:

$$
r(\eta)=e^{L(\eta)}\left(|\eta|^{2}+1\right) .
$$

The transformation $\tau: \eta \rightarrow \zeta$ is obtained by imposing Snell's Law on the reflector and is expressed by

$$
\zeta=\eta+\frac{1}{L_{\eta}}
$$

where $L_{\eta}$ is the derivative of $L$ with respect to $\eta$ [4].

From Conservation of Energy, the relation between the feed radiation and the specified far-field power pattern is given by a nonlinear MA equation of elliptic ( - sign) or hyperbolic (+) type in terms of $L(\eta)$ [4] as follows:

$$
\left|L_{\eta \eta}-L_{\eta}^{2}\right|^{2}-\left|L_{\eta \bar{\eta}}\right|^{2}= \pm \frac{I(\eta)}{G(\zeta)}\left(\frac{1+|\zeta|^{2}}{1+|\eta|^{2}}\right)^{2}
$$

where $L_{\eta \eta}$ is the second partial derivative of $L$ with respect to $\eta$ and $L_{\eta \bar{\eta}}$ is the second partial derivative of $L$ with respect to $\eta$ and its complex conjugate $\bar{\eta}$.

2.1. Reflector Surface Representation. Several iterative numerical techniques have been employed to solve the elliptical type of the MA equation, leading to a discrete representation of the surface at a grid of points. In $[5,7]$ the authors employed a 9point cell finite-difference numerical scheme to approximate the derivatives of $L(\eta)$ in (3) and (4).

Here, alternatively, the numerical solution employs axisdisplaced confocal quadrics to locally describe the reflector's surface, which allows an analytical representation of the derivatives and the simplification of (3). The solution yields a finite set of points on the reflector surface associated to a grid on $\eta\left[\eta_{1,1}, \ldots, \eta_{j, k}, \ldots, \eta_{J, K}\right]$, where $j$ and $k$ describe the node position at the grid. Although the technique could be applied to any type of grid, in this work we adopt a polar grid for comparative purposes, where $J$ and $K$ are the numbers of rings and radials, respectively. The polar grid is defined in terms of $\theta^{\prime}$ and $\phi^{\prime}$ referred to the $z^{\prime}$-axis (feed axis), as shown in Figure 2. The external ring of the polar grid defines the feed cone edge with semiangle $\theta_{c}$. The reflector point associated to the center of the grid is assumed to be 


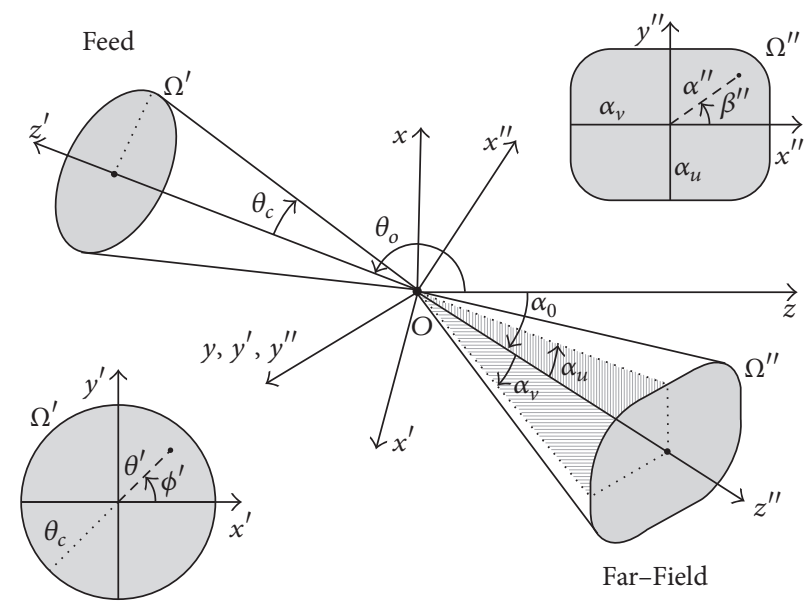

FIGURE 2: Feeder and far-field auxiliary coordinate systems.

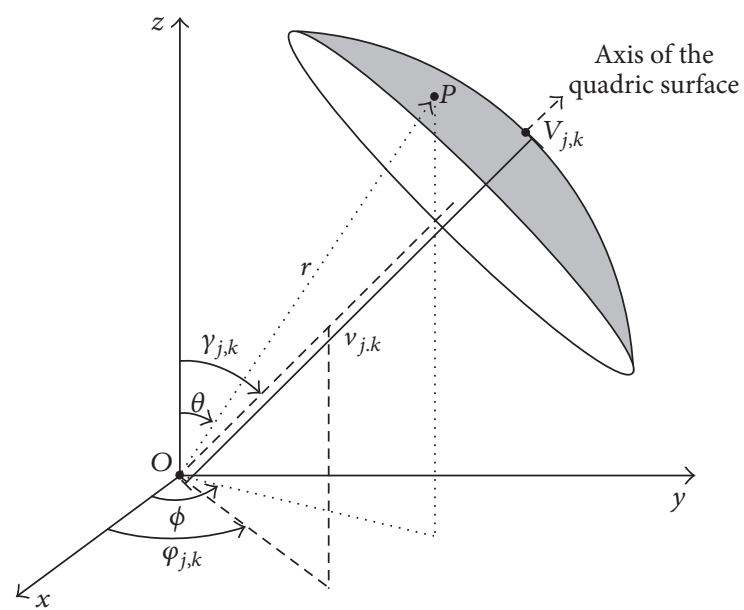

FIgURE 3: Local axis-displaced confocal quadric surface.

known for the numerical solution and can be used in a postsynthesis adjustment of the reflector dimensions, as the $\mathrm{GO}$ formulation involves only derivatives of the real function $L$ employed to represent the reflector surface.

For each ray direction $\eta_{j, k}$ corresponding to node $j, k$ the surface point is defined by $L_{j, k}$ with the help of (2). The quadric $j, k$ that describes the surface around this point, illustrated in Figure 3, is given by [4] as follows:

$$
\begin{aligned}
a_{j, k} e^{-L}= & (\bar{\eta}+\eta) b_{j, k}+i(\bar{\eta}-\eta) c_{j, k}+\left(|\eta|^{2}-1\right) d_{j, k} \\
& -|\eta|^{2}-1,
\end{aligned}
$$

where

$$
\begin{aligned}
a_{j, k} & =-e_{j, k} v_{j, k}, \\
b_{j, k} & =e_{j, k} \sin \gamma_{j, k} \cos \varphi_{j, k}, \\
c_{j, k} & =e_{j, k} \sin \gamma_{j, k} \sin \varphi_{j, k}, \\
d_{j, k} & =e_{j, k} \cos \gamma_{j, k} ;
\end{aligned}
$$

$a_{j, k}$ is the semilatus rectum, $e_{j, k}$ is the eccentricity, $v_{j, k}$ is the distance between $O$ and the quadric vertex $V_{j, k}$, and $\varphi_{j, k}$ and $\gamma_{j, k}$ are the azimuth and elevation angles of the quadric axis (see Figure 3). The four parameters $a_{j, k}, b_{j, k}, c_{j, k}$, and $d_{j, k}$ are calculated by imposing that the quadric matches the reflector at the points associated with the 4-point cell around the grid node $j, k$, as depicted in Figure 4 . This leads to analytical expressions for the parameters involving the values of $L$ at the 4-point cell $\left(L_{j, k}, L_{j-1, k}, L_{j+1, k-1}\right.$, and $\left.L_{j+1, k+1}\right)$. The polar grid in Figure 4 is generated with the help of (1) and incrementing $\theta_{1, k}=0$ to $\theta_{J, k}=\theta_{c}$, with $\theta_{j, k}-\theta_{j-1, k}=\Delta \theta$, and $\phi_{j, 1}=0$ to $\phi_{j, K}=2 \pi-\Delta \phi$, with $\phi_{j, k}-\phi_{j, k-1}=\Delta \phi=$ $2 \pi / K$.

By differentiating (5) with respect to $\eta$ and its conjugate $\bar{\eta}$ and replacing the derivatives of $L$ into (4), one obtains

$$
\begin{aligned}
& \frac{I\left(\eta_{j, k}\right)}{G\left(\zeta_{j, k}\right)}\left(\frac{\left|\zeta_{j, k}\right|^{2}+1}{\left|\eta_{j, k}\right|^{2}+1}\right)^{2} \\
& \quad=\left|\frac{b_{j, k}^{2}+c_{j, k}^{2}+d_{j, k}^{2}-1}{\left[\left(d_{j, k}-1\right) \bar{\eta}+\left(b_{j, k}-i c_{j, k}\right)\right]^{2}}\right|^{2},
\end{aligned}
$$

where Snell's Law on the reflector imposes the relation between $\eta_{j, k}$ and $\zeta_{j, k}$, expressed by the following equation [4]:

$$
\zeta_{j, k}=\frac{\left(d_{j, k}+1\right)-\left(b_{j, k}+i c_{j, k}\right) \bar{\eta}_{j, k}}{\left(b_{j, k}-i c_{j, k}\right)+\left(d_{j, k}-1\right) \bar{\eta}_{j, k}} .
$$

It should be noted that, for a quadric, $\left|L_{\eta \eta}-L_{\eta}^{2}\right|=0$, yielding the simplification of (4). 


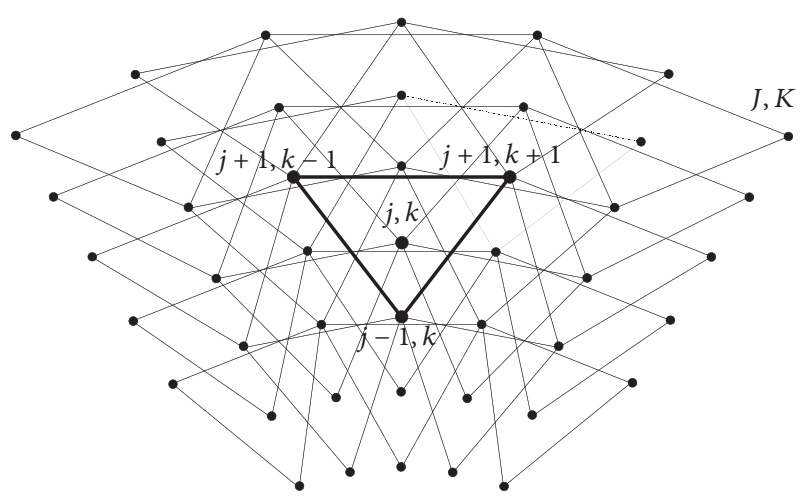

FIGURE 4: Node scheme for the inner 4-point cells.

From (7) it is possible to define the operator $\Gamma_{j, k}$ for all inner nodes of the grid as follows:

$$
\begin{aligned}
\Gamma_{j, k}= & -\frac{I\left(\eta_{j, k}\right)}{G\left(\zeta_{j, k}\right)}\left(\frac{\left|\zeta_{j, k}\right|^{2}+1}{\left|\eta_{j, k}\right|^{2}+1}\right)^{2} \\
& +\left|\frac{b_{j, k}^{2}+c_{j, k}^{2}+d_{j, k}^{2}-1}{\left[\left(d_{j, k}-1\right) \bar{\eta}_{j, k}+\left(b_{j, k}-i c_{j, k}\right)\right]^{2}}\right|^{2}
\end{aligned}
$$

which represents a nonlinear equation in terms of the values of $L$ in the 4-point cell $\left(L_{j, k}, L_{j-1, k}, L_{j+1, k-1}\right.$, and $\left.L_{j+1, k+1}\right)$ centered at node $j, k$. For $j=1, L_{j-1, k}$ corresponds to the central point of the surface, that is defined prior to the numerical solution and is kept constant along the iterations.

2.2. Boundary Conditions. To apply the synthesis technique, we consider that the feed source radiates a conical tube of rays with semivertex angle $\theta_{c}$ and axis tilted by $\theta_{o}$ from the $z$-axis, as illustrated in Figure 2. After reflection, the rays are confined within a solid angle with perimeter $\Omega^{\prime \prime}$ in the plane $\zeta^{\prime \prime}$ given by [4] as follows:

$$
\zeta^{\prime \prime}=\frac{\zeta_{o} \zeta-1}{\zeta_{o}-\zeta} .
$$

In the examples investigated in Section 3, we have considered an approximately square perimeter $\Omega^{\prime \prime}$ described by a superellipse as follows:

$$
\begin{aligned}
& \left|\frac{1}{2 \zeta_{u}}\left(\frac{\zeta_{o} \zeta+1}{\zeta_{o}-\zeta}+\frac{\zeta_{o} \bar{\zeta}+1}{\zeta_{o}-\bar{\zeta}}\right)\right|^{2 \sigma} \\
& \quad+\left|\frac{1}{2 i \zeta_{v}}\left(\frac{\zeta_{o} \zeta+1}{\zeta_{o}-\zeta}-\frac{\zeta_{o} \bar{\zeta}+1}{\zeta_{o}-\bar{\zeta}}\right)\right|^{2 \sigma}=1
\end{aligned}
$$

As $\sigma \rightarrow 1, \Omega^{\prime \prime}$ tends to be an ellipse with axes $2 \zeta_{u}=$ $\cot \left(\alpha_{u} / 2\right)$ and $2 \zeta_{v}=\cot \left(\alpha_{v} / 2\right)$, and center at $\zeta_{o}=\cot \left(\alpha_{o} / 2\right)$, where $2 \alpha_{u}$ and $2 \alpha_{v}$ are the far-field beamwidths in the vertical and horizontal planes; respectively, and $\alpha_{o}$ is the beam offset angle with respect to the $z$-axis, as shown in Figure 2. As $\sigma \rightarrow \infty, \Omega^{\prime \prime}$ tends to be a rectangle with sides $2 \zeta_{v}$ and $2 \zeta_{u}$.

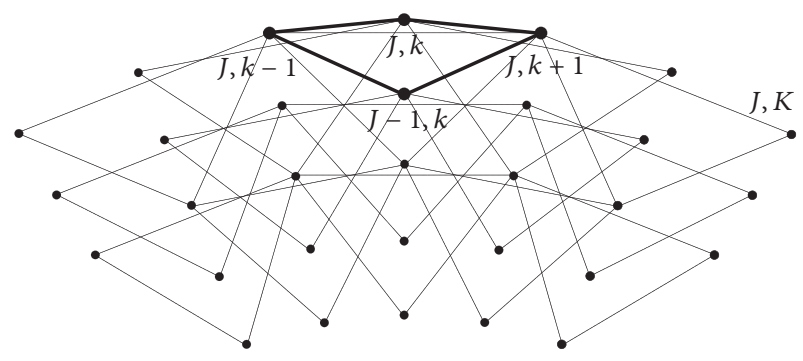

Figure 5: Node scheme for the boundary 4-point cells.

The boundary condition is imposed by enforcing the rays at the feed cone perimeter $\Omega^{\prime}$ to map into the far-field superelliptical perimeter $\Omega^{\prime \prime}$ after reflection. For the numerical solution, at each ray direction $\eta_{J, k}$ corresponding to a boundary node $J, k$ (see Figure 5 ), the real function $L$ is approximated by a quadric given by (5). The parameters $a_{J, k}$, $b_{J, k}, c_{J, k}$, and $d_{J, k}$ are calculated by imposing that the quadric passes at the reflector points associated with the 4-point cell around node $J, k$, as depicted in Figure 5. This leads to analytical expressions for the four parameters in terms of the values of $L$ at the cell nodes $\left(L_{J, k}, L_{J-1, k}, L_{J, k-1}\right.$, and $\left.L_{J, k+1}\right)$.

Once the expressions for the parameters are found, the mapping $\tau: \eta_{J, k} \rightarrow \zeta_{J, k}$ can be expressed with the help of (8) and replaced into (11). It allows one to define an operator for the grid nodes at the perimeter $\Omega^{\prime}$ as

$$
\begin{aligned}
\Gamma_{J, k}= & -1+\left|\frac{1}{2 \zeta_{u}}\left(\frac{\zeta_{o} \zeta_{J, k}+1}{\zeta_{o}-\zeta_{J, k}}+\frac{\zeta_{o} \bar{\zeta}_{J, k}+1}{\zeta_{o}-\bar{\zeta}_{J, k}}\right)\right|^{2 \sigma} \\
& +\left|\frac{1}{2 i \zeta_{V}}\left(\frac{\zeta_{o} \zeta_{J, k}+1}{\zeta_{o}-\zeta_{J, k}}-\frac{\zeta_{o} \bar{\zeta}_{J, k}+1}{\zeta_{o}-\bar{\zeta}_{J, k}}\right)\right|^{2 \sigma} .
\end{aligned}
$$

Equation (12) represents a nonlinear equation in terms of $L$ at the 4-point cell around node $J, k$.

2.3. Numerical Iterative Scheme. The application of (9) or (12) at each grid node leads to a system of $J \times K$ nonlinear equations in terms of $L\left[L_{1,1}, \ldots, L_{j, k}, \ldots, L_{J, K}\right]$. For the numerical solution of the system of nonlinear equations, Newton's method has been employed [4-7]. It is based on an iterative scheme where convergence and stability depend on the choice of an adequate initial solution for the reflector's surface. In this work, a single quadric was adopted as initial solution at all grid nodes, chosen to approximate the farfield beamwidth in the vertical plane. The iterative scheme continues until $\left|\Gamma_{j, k}\right|<\varepsilon$ at all nodes, where $\varepsilon$ is a specified maximum value for the operator residues.

\section{Design Examples}

To illustrate the numerical scheme described in Section 2, two examples of offset shaped reflector antennas are presented. They are shaped to provide, according to GO principles, a Gaussian far-field radiation pattern $G(\zeta)$ in a solid angle 


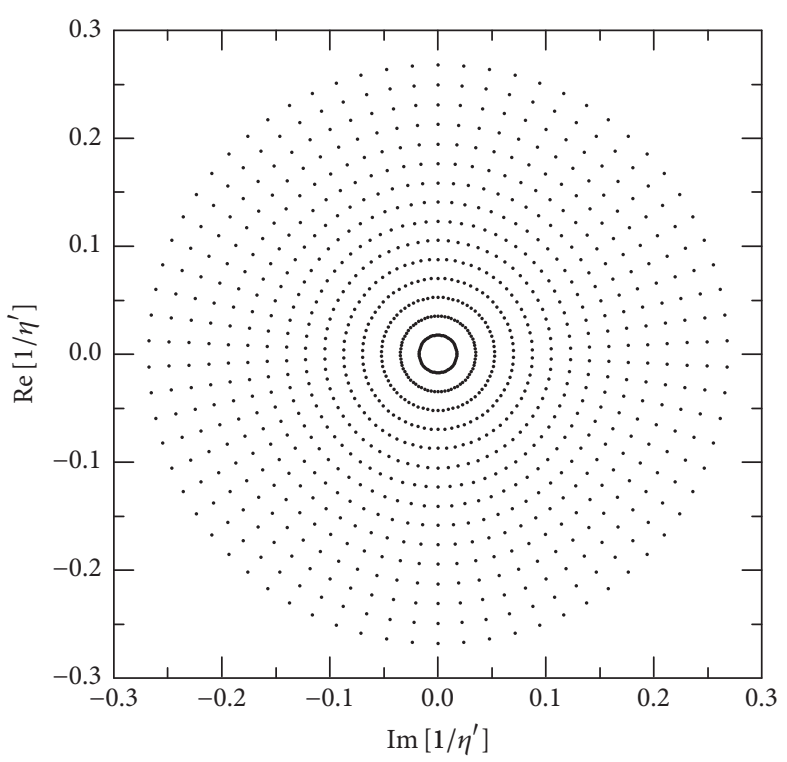

(a)

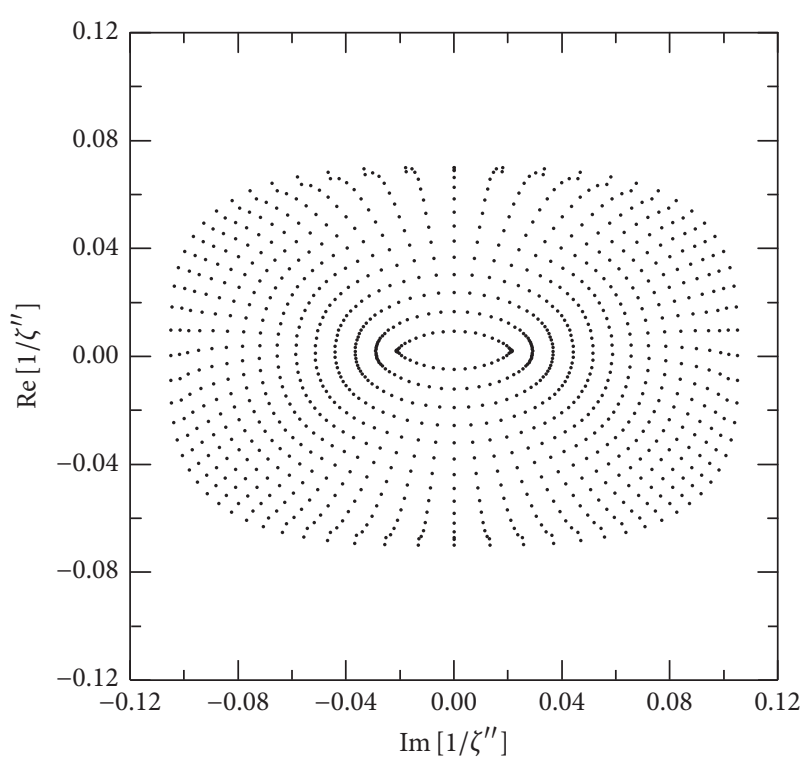

(b)

FIgURE 6: Uniform grid representation of the ray directions at the (a) feed (complex plane $\eta^{\prime}$ ) and (b) far-field (complex plane $\zeta^{\prime \prime}$ ).

defined by a superelliptical perimeter $\Omega^{\prime \prime}$ in the $\zeta^{\prime \prime}$ plane and described by

$$
G(\zeta)=G_{0} e^{-g\left|\rho^{\prime \prime}\right|},
$$

where the parameter $g$ is chosen to specify the attenuation at the far-field boundary, requiring equal coverage at the superelliptical contour defined by $\rho^{\prime \prime}$ as follows:

$$
\begin{aligned}
\rho^{\prime \prime}= & \left|\frac{1}{2 \zeta_{u}}\left(\frac{\zeta_{o} \zeta+1}{\zeta_{o}-\zeta}+\frac{\zeta_{o} \bar{\zeta}+1}{\zeta_{o}-\bar{\zeta}}\right)\right|^{2 \sigma} \\
& +\left|\frac{1}{2 i \zeta_{v}}\left(\frac{\zeta_{o} \zeta+1}{\zeta_{o}-\zeta}-\frac{\zeta_{o} \bar{\zeta}+1}{\zeta_{o}-\bar{\zeta}}\right)\right|^{2 \sigma} .
\end{aligned}
$$

For both examples, the parameter $g=1.382$ is set to provide $6 \mathrm{~dB}$ attenuation at the contour $\Omega^{\prime \prime}$. A feed with a raisedcosine model $I(\eta)=I_{0} \cos ^{2 n} \theta^{\prime}$ illuminates the reflector antenna, with $n=9.605$ to provide $12 \mathrm{~dB}$ attenuation at the reflector edge. The feed cone has an offset angle $\theta_{o}=130^{\circ}$ and half-angle $\theta_{c}=30^{\circ}$ (see Figure 2 ) and it is vertically polarized.

For the first example, the far-field coverage region is specified by $\alpha_{o}=-18^{\circ}, \alpha_{u}=8^{\circ}, \alpha_{v}=12^{\circ}$, and $\sigma=1.6$ (see Figure 2). The initial solution $L^{(0)}$ for the iterative numerical scheme is an ellipsoid with $a^{(0)}=-40.893 \mathrm{~cm}, b^{(0)}=$ $-0.077795, c^{(0)}=0$, and $d^{(0)}=0.62708$, which provides a beamwidth equal to the specification in the symmetry plane, $\alpha_{u}=8^{\circ}$. To ensure convergence, the far-field perimeter $\Omega^{\prime \prime}$ is gradually deformed from a circle to the specified superelliptical perimeter. For the synthesis, we first employed a regular polar grid with $J=15$ rings, $K=70$ radials (i.e., 980 interior nodes and 70 boundary nodes), and center at the feed axis, as illustrated in Figure 6(a). Consequently, 1,050 local quadrics were used to attain the shaped reflector. A total of $m=16$ iterations were necessary to obtain $\left|\Gamma_{j, k}\right|<$ $\varepsilon=10^{-5}$ at all grid nodes, where $|\Gamma|_{\text {mean }}=7.683 \times 10^{-8}$. During the iterative process, we have observed that the largest residues occur close to the reflector's edge, probably due to the superelliptical perimeter imposed at $\Omega^{\prime \prime}$. The shaped reflector has approximately an elliptical projection in $x y$ plane. By adding a proper constant to the final solution $L(\eta)$, the projected diameters in the symmetry plane $(\phi=$ 0 ) and orthogonal plane are $25 \mathrm{~cm}$ and $29 \mathrm{~cm}$, respectively. Consequently, the distance from $O$ to the final reflector along the ray at center of the polar grid becomes $r_{0}=27.958 \mathrm{~cm}$. Figures $6(\mathrm{a})$ and $6(\mathrm{~b})$ illustrate the grids resulting from the synthesis in the complex planes $\eta^{\prime}$ and $\zeta^{\prime \prime}$, respectively, for the initial and final reflectors.

As observed in Figure 6(b), the superelliptical perimeter is achieved at $\Omega^{\prime \prime}$. To compensate for the feed attenuation $(12 \mathrm{~dB})$ at the reflector edge, the synthesis moves far-field rings closer to each other toward the coverage perimeter. The increase in the concentration of points indicates the reflector area where the shaping is more intense when compared with the initial solution and some distortion may be observed in areas close to the symmetry plane $\operatorname{Im}\left[1 / \zeta^{\prime \prime}\right]=0$. On the other hand, the regular polar grid intrinsically generates a denser grid of points at the most inner ring along the asymmetry plane which may bring some numerical instabilities along the iterative procedure. As mentioned before, the numerical scheme described in Section 2 presents flexibility to handle different spacings in the polar grid. To compensate these effects and provide a better description of the reflector surface, we alternatively employed a nonuniform polar grid in the $\eta^{\prime}$ plane where the spacing between the rings follows approximately a Gaussian rule with larger $\Delta \theta$ at the center and shorter one at the outer rings, thus reducing the density 


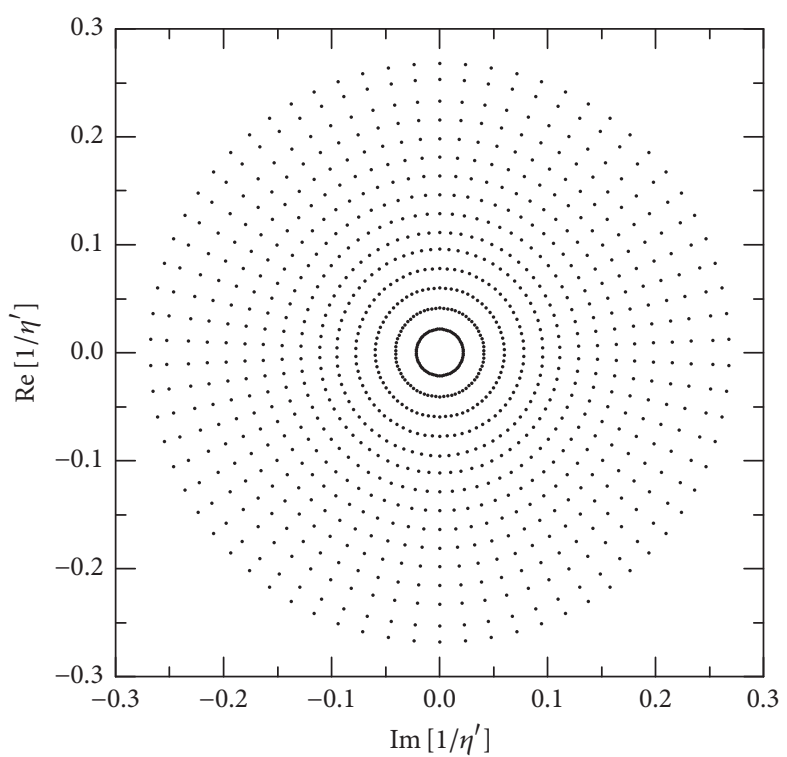

(a)

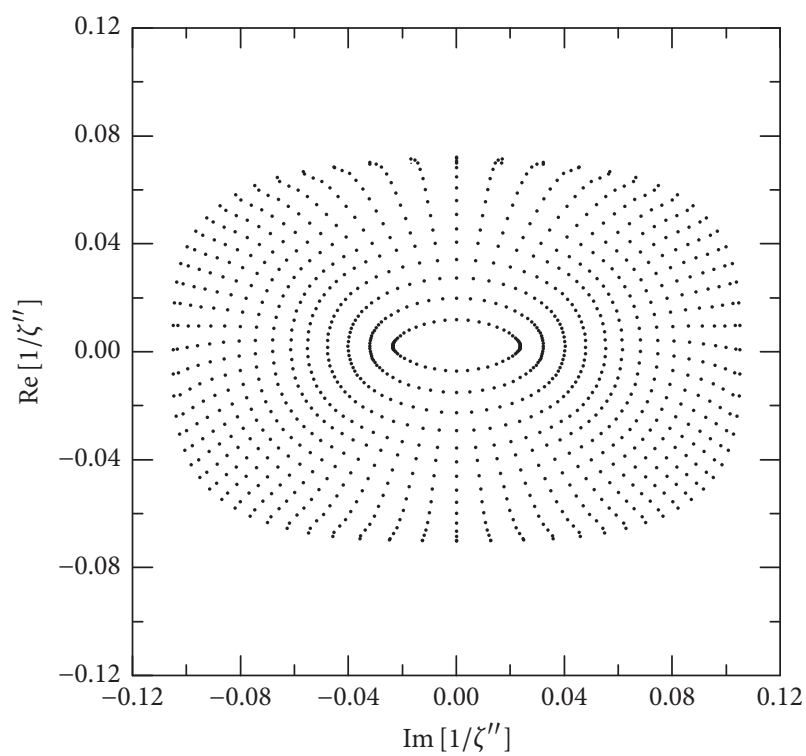

(b)

FiguRE 7: Nonuniform polar grid representation of the ray directions at the (a) feed (complex plane $\eta^{\prime}$ ) and (b) far-field (complex plane $\zeta^{\prime \prime}$ ).

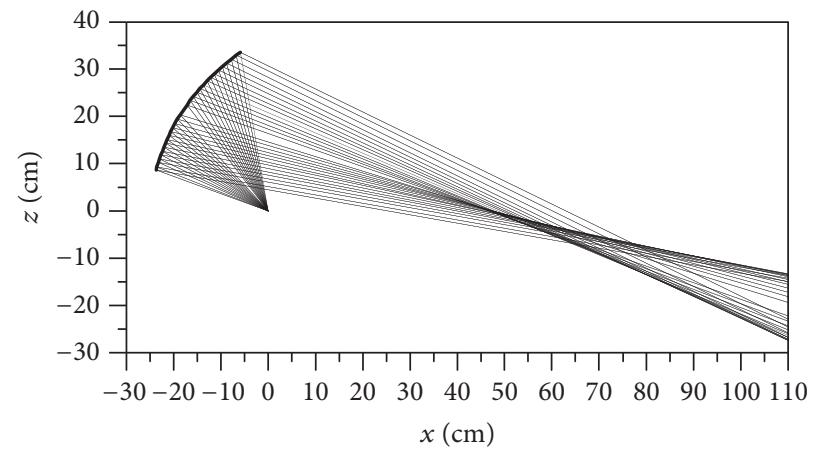

FIGURE 8: Shaped reflector surface and rays at the symmetry plane $(\phi=0)$ of the first example.

of points at the center and increasing it close to the boundary [8]. Figures 7(a) and 7(b) present the new distributions of grid points in the complex planes $\eta^{\prime}$ and $\zeta^{\prime \prime}$, respectively, and, as one can observe, the mapped points in the plane $\zeta^{\prime \prime}$ show a more coherent distribution, especially close to the boundary and the symmetry plane.

Figure 8 shows a cut in the symmetry plane of the final reflector and the corresponding rays obtained from the nonuniform grid arrangement of Figure 7. To validate the results, the shaped reflector was analysed by Physical Optics (PO) with equivalent edge currents [11]. To obtain a smooth description of the reflector, the reflector points obtained from the GO shaping were interpolated by a polynomial plus Fourier series [12]. Figure 9 compares the GO and PO radiation patterns in the symmetry plane $(\phi=0)$ at 28 and $38 \mathrm{GHz}$. A good agreement was achieved and the differences observed in Figure 9 can be attributed to diffractive effects not taken into account by the GO synthesis. Figures 10(a) and 10 (b) show the contour plots in the $u v$-plane of the PO radiation patterns at $28 \mathrm{GHz}$ and $38 \mathrm{GHz}$, respectively, which are compared with the desired GO superelliptical beam contour described by the dashed contour.

In the second design example, the far-field coverage region has a superelliptical contour, with the longer axis along the symmetry plane, that is, $\alpha_{u}=12^{\circ}$ and $\alpha_{v}=8^{\circ}$. Different from the first case, now the stretching of the farfield grid is more intense in the asymmetry plane close to the boundary. The initial solution $L^{(0)}$ for the iterative numerical scheme is an ellipsoid with $a^{(0)}=-37.469 \mathrm{~cm}, b^{(0)}=$ $-0.007115, c^{(0)}=0$, and $d^{(0)}=0.510939$, which provides a beamwidth coverage equal to the specified in the symmetry plane with $\alpha_{u}=12^{\circ}$. A total of $m=15$ iterations were necessary to obtain $\left|\Gamma_{j, k}\right|<\varepsilon=10^{-5}$ at all grid nodes, where $|\Gamma|_{\text {mean }}=3.706 \times 10^{-8}$. Figure 11 illustrates the shaped reflector and the rays from the feed point source at the origin to the far-field in the symmetry plane. The shaped reflector has approximately an elliptical projection in plane $x y$ and, by 


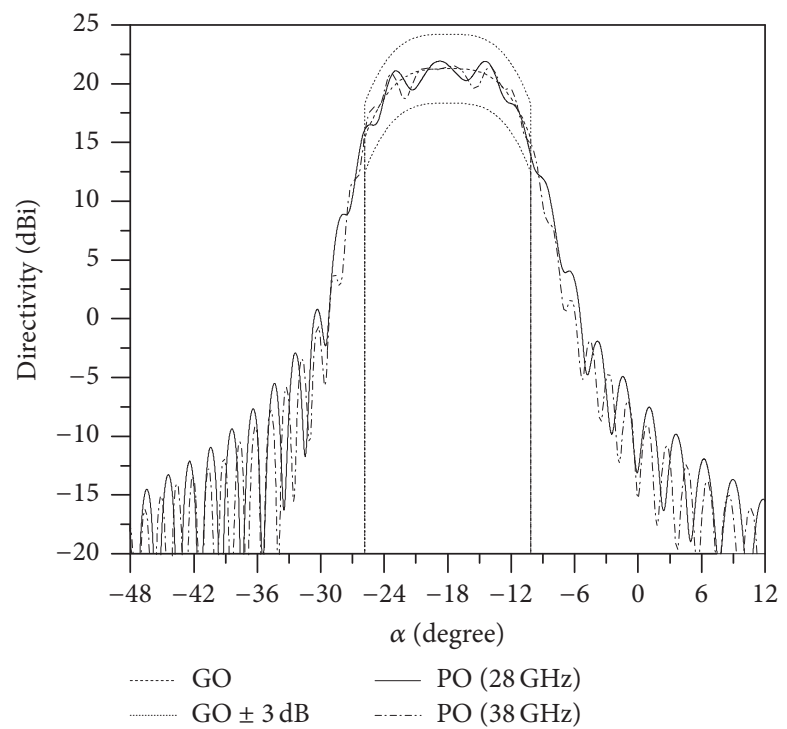

FIGURE 9: Radiation patterns in the symmetry plane $(\phi=0)$ of the first example.

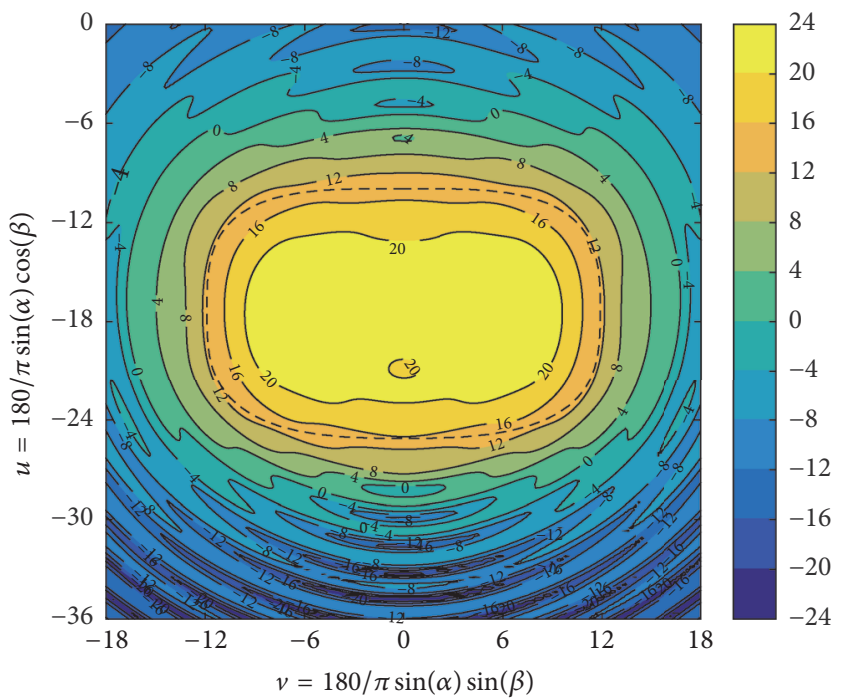

(a)

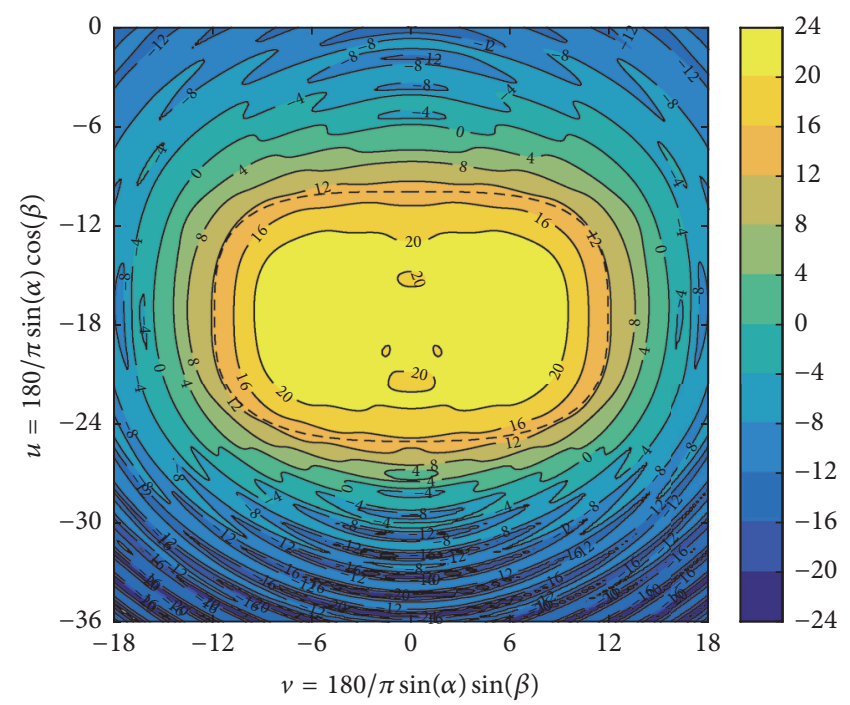

(b)

FIGURE 10: Radiation pattern (dBi) in the $u v$-plane at (a) $28 \mathrm{GHz}$ and (b) $38 \mathrm{GHz}$ of the first example.

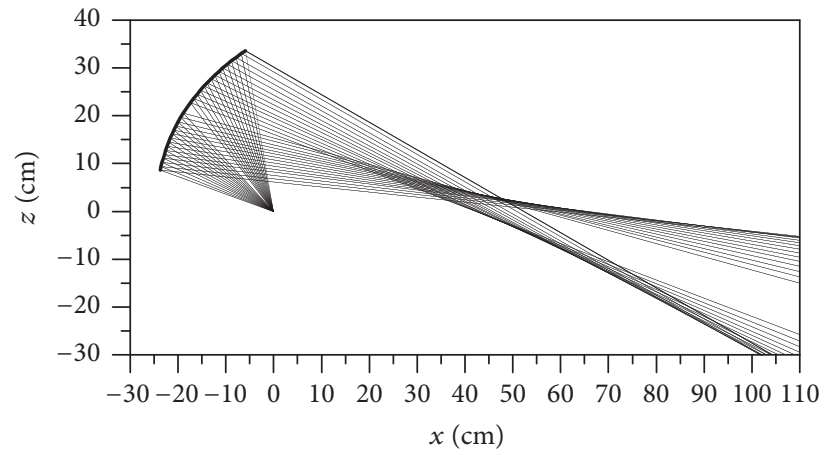

FiguRE 11: Shaped reflector surface and rays at the symmetry plane $(\phi=0)$ of the second example. 


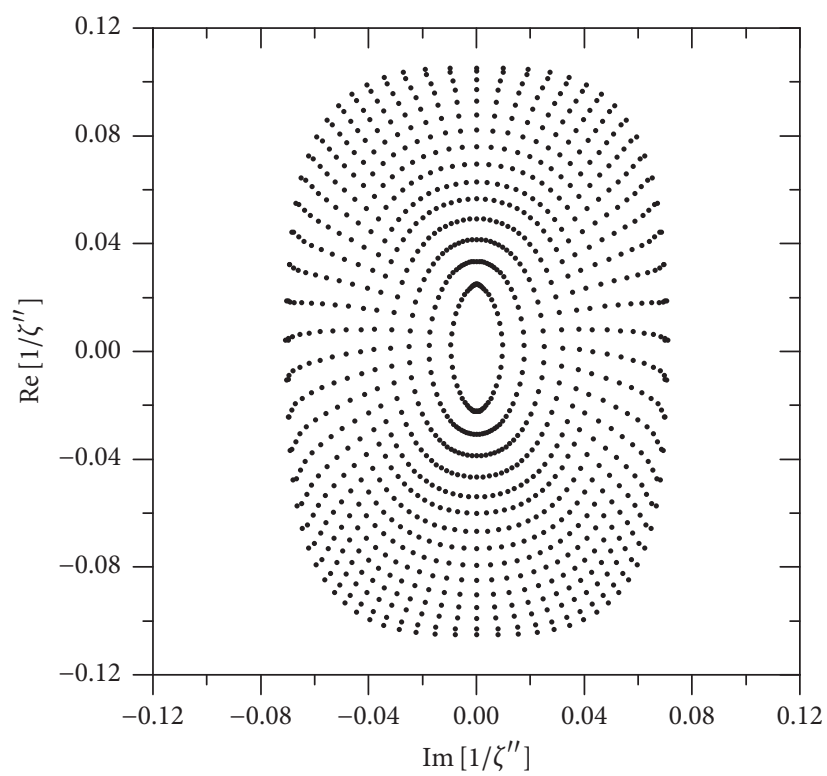

FIGURE 12: Representation of the ray directions at the far-field (complex plane $\zeta^{\prime \prime}$ ) of the second example.

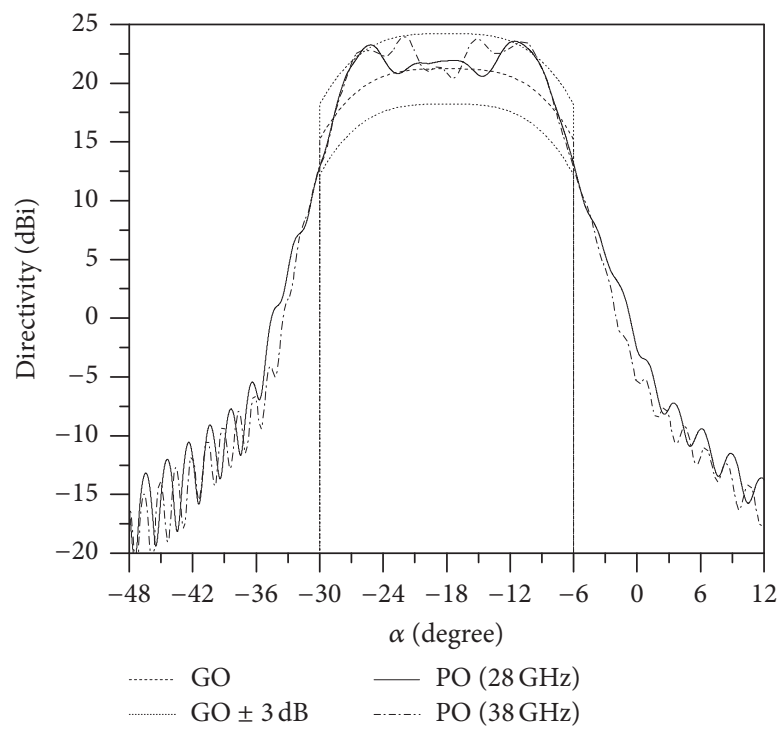

FIGURE 13: Radiation patterns in the symmetry plane $(\phi=0)$ of the second example.

adding a constant to the synthesized solution $L(\eta)$, the diameter in the symmetry plane is again adjusted to $25 \mathrm{~cm}$, yielding the diameter of $29.75 \mathrm{~cm}$ in the asymmetry plane and the distance $r_{0}=28.32 \mathrm{~cm}$ from $O$ to the reflector along the ray at center of the polar grid. For this example, the same nonuniform grid in the complex plane $\eta^{\prime}$ was employed (as illustrated in Figure $7(\mathrm{a})$ ) and the resulting mapping in the complex plane $\zeta^{\prime \prime}$ is illustrated in Figure 12.

Once again, the shaped reflector was interpolated with a polynomial plus Fourier series and analysed by PO [11, 12]. Figure 13 shows the $\mathrm{GO}$ and $\mathrm{PO}$ radiation patterns in the symmetry plane $(\phi=0)$ at $28 \mathrm{GHz}$ and $38 \mathrm{GHz}$. A good agreement between the desired $\mathrm{GO}$ and $\mathrm{PO}$ radiation pattern results is observed. Figures $14(\mathrm{a})$ and $14(\mathrm{~b})$ show the PO radiation pattern at $28 \mathrm{GHz}$ and $38 \mathrm{GHz}$, respectively, in the $u v$-plane, where one observes the desired superelliptical beam contour achieved for the main lobe (the dashed contour represents the desired GO coverage).

\section{Conclusions}

This work presented an alternative numerical scheme for the solution of the MA equation resulting from the exact formulation of the GO synthesis of offset reflector antennas. It explores the local interpolating properties of confocal quadrics to represent shaped reflector surfaces, which allows 


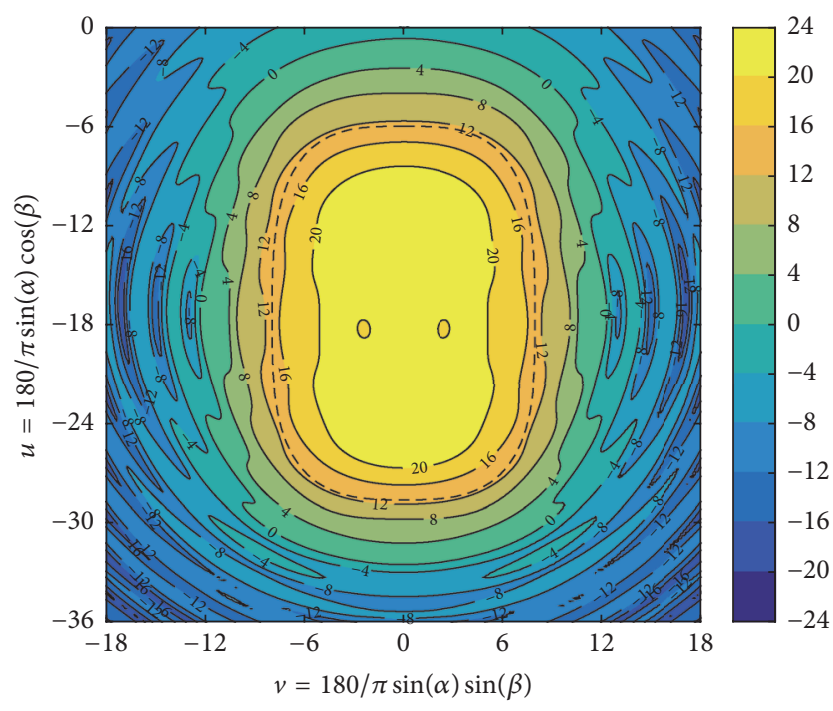

(a)

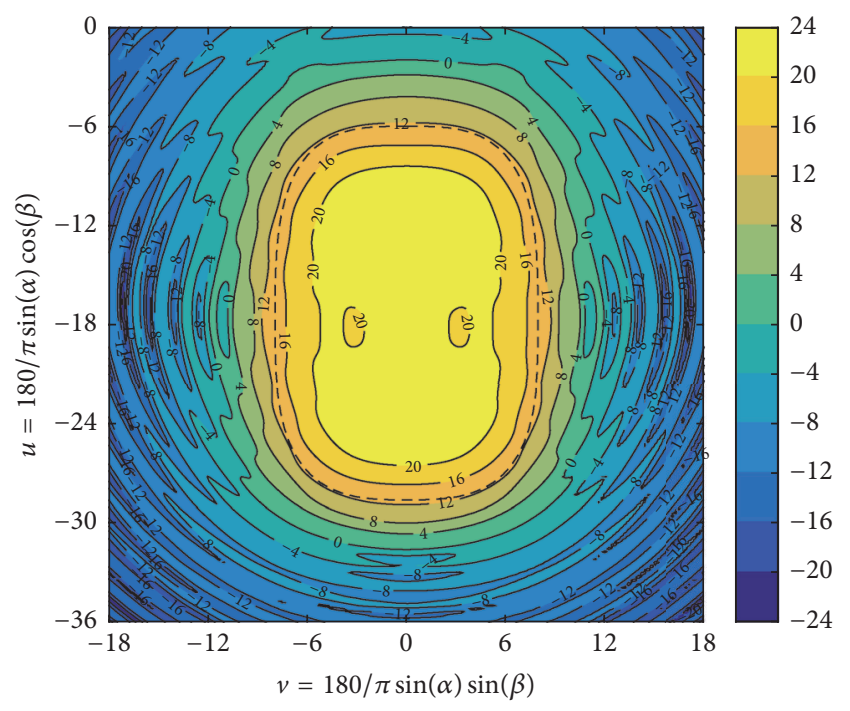

(b)

Figure 14: Radiation pattern (dBi) in the $u v$-plane at (a) $28 \mathrm{GHz}$ and (b) $38 \mathrm{GHz}$ of the second example.

the partial derivatives involved in the formulation to be analytically expressed and provides a significant simplification of the MA equation. The numerical scheme was used to shape two reflectors radiating Gaussian patterns within superelliptical cones. The shaped reflectors were analysed by the PO approximation with equivalent edge currents and the results showed good agreement with the GO radiation pattern specifications. The method proved to be very fast and stable, achieving convergence with relatively few iterations. This behaviour is directly linked with the analytical determination of the derivatives $\partial \Gamma_{j, k}^{(m)} / \partial L_{p, q}$. Different from the finite-difference approach, the present method can be extended to other types of grids and contours $\Omega^{\prime}$ with minor efforts.

\section{Competing Interests}

The authors declare that they have no competing interests.

\section{References}

[1] J. S. Schruben, "Formulation of a reflector-design problem for a lighting fixture," Journal of the Optical Society of America, vol. 62, no. 12, pp. 1498-1501, 1972.

[2] F. Brickell, L. Marder, and B. S. Westcott, "The geometrical optics design of reflectors using complex coordinates," Journal of Physics A: General Physics, vol. 10, no. 2, pp. 245-260, 1977.

[3] F. Brickell and B. S. Westcott, "Phase and power density distributions on plane apertures of reflector antennas," Journal of Physics. A. Mathematical and General, vol. 11, no. 4, pp. 777789, 1978.

[4] B. S. Westcott, F. A. Stevens, and F. Brickell, "GO synthesis of offset dual reflectors," IEE Proceedings H: Microwaves, Optics and Antennas, vol. 128, no. 1, pp. 11-18, 1981.

[5] M. Mehler, S. Tun, and N. Adatia, "Direct far-field GO synthesis of shaped beam reflector antennas," IEE Proceedings $H$ :
Microwaves, Antennas and Propagation, vol. 133, no. 3, pp. 213220, 1986.

[6] J. Bergmann, R. C. Brown, P. J. B. Clarricoats, and H. Zhou, "Synthesis of shaped-beam reflector antenna patterns," IEE Proceedings H: Microwaves, Antennas and Propagation, vol. 135, no. 1, pp. 48-53, 1988.

[7] B. Chantraine-Barès, R. Sauleau, L. Le Coq, and K. Mahdjoubi, "A new accurate design method for millimeter-wave homogeneous dielectric substrate lens antennas of arbitrary shape," IEEE Transactions on Antennas and Propagation, vol. 53, no. 3, pp. 1069-1082, 2005.

[8] P.-S. Kildal, "Synthesis of multireflector antennas by kinematic and dynamic ray tracing," IEEE Transactions on Antennas and Propagation, vol. 38, no. 10, pp. 1587-1599, 1990.

[9] F. J. S. Moreira and J. R. Bergmann, "Shaping axis-symmetric dual-reflector antennas by combining conic sections," IEEE Transactions on Antennas and Propagation, vol. 59, no. 3, pp. 1042-1046, 2011.

[10] R. A. Penchel, J. R. Bergmann, and F. J. Moreira, "Main-reflector shaping of omnidirectional dual reflectors using local conic sections," IEEE Transactions on Antennas and Propagation, vol. 61, no. 8, pp. 4379-4383, 2013.

[11] A. Michaeli, "Equivalent edge currents for arbitrary aspects of observation," IEEE Transactions on Antennas and Propagation, vol. 32, no. 3, pp. 252-258, 1984.

[12] J. R. Bergmann, F. J. V. Hasselmann, F. L. Teixeira, and C. G. Rego, "Comparison between techniques for global surface interpolation in shaped reflector analysis," IEEE Transactions on Antennas and Propagation, vol. 42, no. 1, pp. 47-53, 1994. 


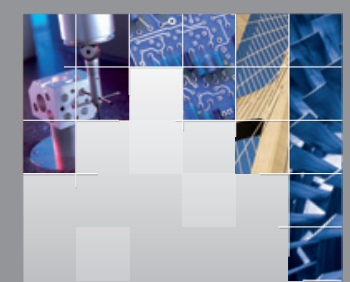

\section{Enfincering}
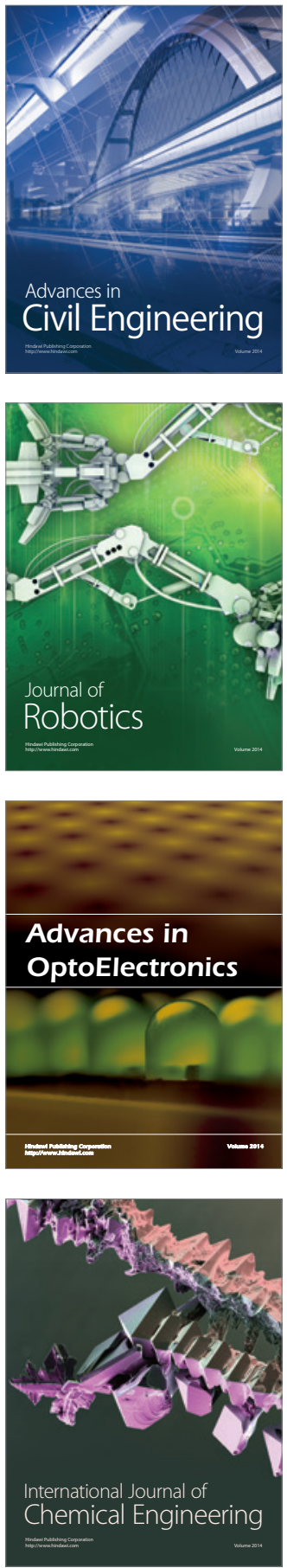

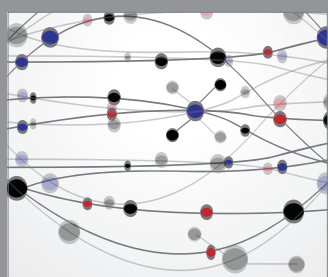

The Scientific World Journal

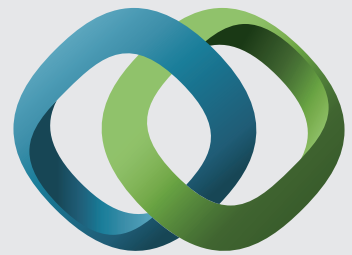

\section{Hindawi}

Submit your manuscripts at

http://www.hindawi.com
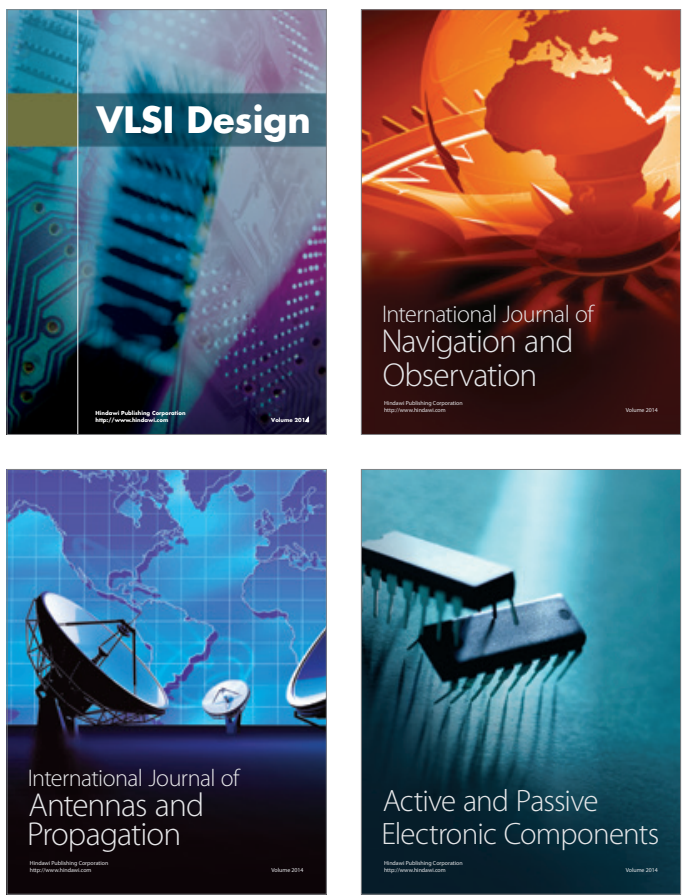
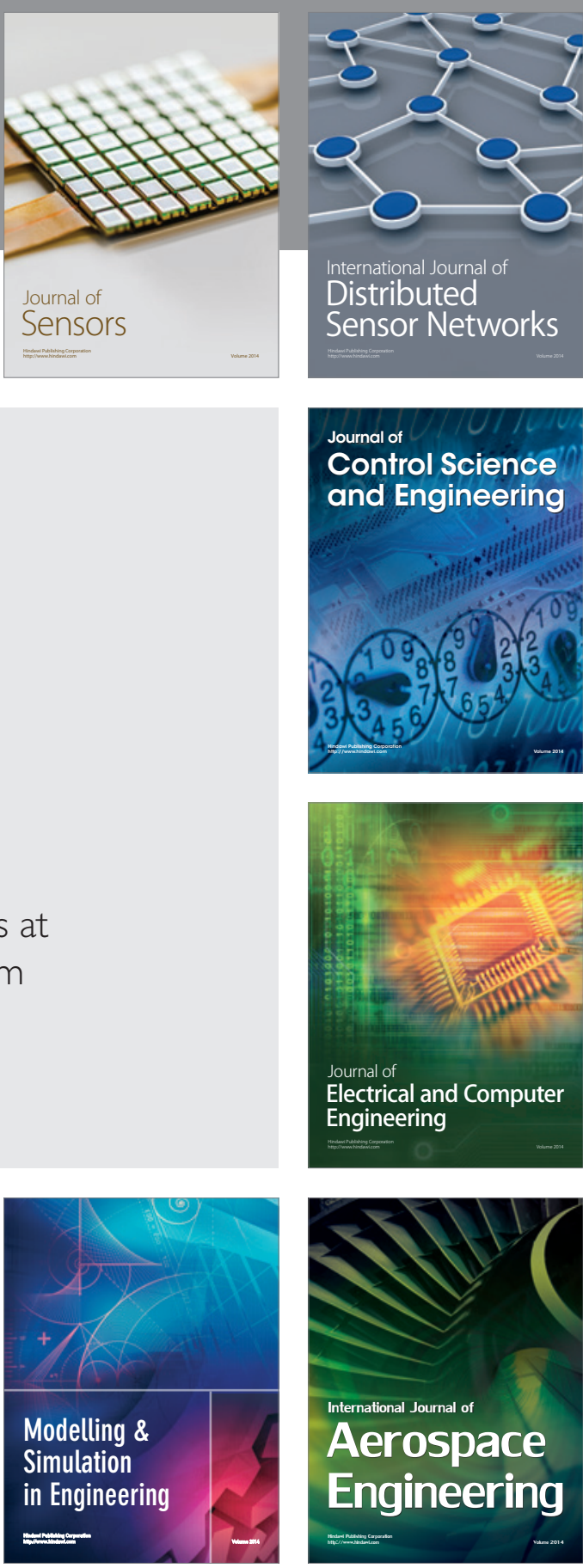

International Journal of

Distributed

Sensor Networks

Journal of

Control Science

and Engineering
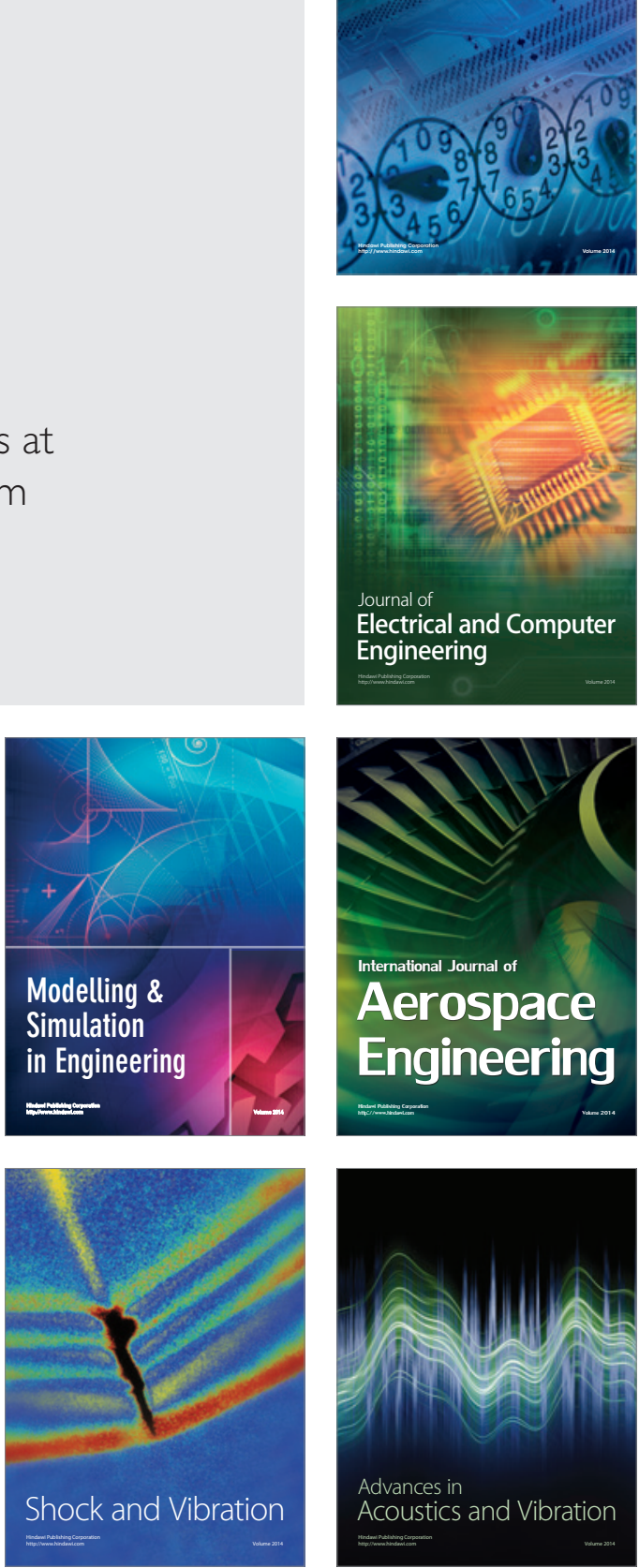